International Journal of Wireless \& Mobile Networks (IJWMN) Vol. 4, No. 4, August 2012

\title{
A SWARM INSPIRED MULTIPATH DATA TRANSMISSION WITH CONGESTION CONTROL IN MANETs using Probabilistic APPROACH
}

\author{
Subhankar joardar $^{1}$, Vandana Bhattacherjee ${ }^{2}$ and Debasis Giri ${ }^{1}$ \\ ${ }^{1}$ Department of Computer Science and Engineering, Haldia Institute of Technology \\ ,Haldia, West Bengal, India \\ subhankarranchi@yahoo.co.in, debasis_giri@hotmail.com \\ ${ }^{2}$ Department of Computer Science and Engineering, Birla Institute of Technology \\ ,Mesra, Jharkhand, India \\ vbhattacharya@bitmesra.ac.in
}

\begin{abstract}
The major causes of network congestion are the lack of network resources and the irrational allocation of network resources. The traditional congestion control methods such as rate control, window mechanism, queue control and others can solve the congestion problem, but for the congestion due to irrational allocation fundamental solution is to make more effectively use of the network resources by adjusting the traffic routing depending on choice may be probabilistic, when congestion occurs. In mobile ad hoc networks congestion creates delay in transmission and also loss of the packet that causes wastage of time and energy on recovery. The Wireless Networks have to play an important role to adopt and execute a large no of innovative application. New challenges have come considering the major limitations of the ad hoc network like node's limited processing power, balance the load of network (to maintain the computation of the node). To overcome the above problem some algorithm is invoked and there may be huge amount of packet loss and this leads to decrease the lifetime of the network. Based on the concept of evolutionary cooperation in swarm Ant, we use the ants swarm intelligence to reinforce good quality routes. The objective of our proposed algorithm is to identify the congestion areas between source and its neighboring nodes to the destination and thus it will help to avoid the congestion of the network in the intermediate links and also minimize the packet loss in the network. Using a new mathematical model considering the swarm-based ant intelligence concept, we found an efficient congestion control mechanism (Ant's probabilistic transition rule).
\end{abstract}

\section{KEYWORDS}

Ad-Hoc Networks, Conditional Probability, Congestion, Distance Vector, Swarm Intelligence, Transmission Quеuе Length.

\section{INTRODUCTION}

Mobile Ad hoc Networks are movable and also able to communicate within themselves using a wireless physical medium where there is no need of pre existing network infrastructure. These networks can form stand-alone groups of wireless terminals, which can be again a part of any other cellular system or a fixed network. The main characteristic which draws the attention is that they are able to configure themselves without a centralized administration.

This paper is based on "A Swarm inspired Probabilistic Path Selection with Congestion Control in MANETs " by S.joardar, V.Bhattacherjee, D.Giri which appeared in the proceedings of second international conference on Computer Science, Engineering And Application, Springer-Verlag, Vol.2, pp 617- 626, May 25-27, 2012

DOI : 10.5121/ijwmn.2012.4408 
International Journal of Wireless \& Mobile Networks (IJWMN) Vol. 4, No. 4, August 2012

The Mobile ad hoc network has several drawbacks that are not found in fixed networks. Unlike in the traditional wireless networks, communication in such a decentralized network is typically multi-hop, with the nodes using each other as relay routers without any fixed infrastructure.

This kind of network is very flexible and suitable for applications such as temporary information sharing in a conference, disaster rescue etc.

However, multi-hop routing, random movement of mobile nodes and other features in MANETs lead to The frequent change in network topology due to the mobility of the nodes causes a great deal of control information onto the network. The small capacity of batteries and the bandwidth limitation of wireless channels are other factors. The scalability is also a major factor because the network performance degrades quickly as the no of nodes increases.

\subsection{Routing in Mobile Ad-hoc Wireless Networks}

Specially configured routing protocols are engaged in order to establish routes between nodes which are more than a single hop. The ability to trace routes in spite of a dynamic topology is the unique feature of these protocols. These protocols can be categorized into two main types: Reactive (On-demand) and Proactive (Table-driven). Evaluating the routes continuously within the network is done by proactive protocols, so when a packet needs to be forwarded the route is already known and can be immediately used. Reactive protocols appeal to a route determination procedure on demand only.

\subsection{Congestion in Mobile Ad-hoc Wireless Networks}

The network where the resources are shared, to minimize the network overload the data rate must be adjusted used by the sender. The packets are dropped due to some routers are not able to forward the packets, so due to the bottleneck condition leads to many packet drops. May be the dropped packets already travelled a long way in the network and thus consumed significant amount of resources. So congestion leads to decrease the network throughput. This type of situation occurred on the early Internet, the development of the TCP congestion control mechanism.

In mobile ad hoc networks congestion occurs due to the shared wireless channel and dynamic topology, packet transmissions suffer from interference and fading. The network load is burdened through the transmission errors. There is an increasing demand of multimedia communications in MANETs so large amount of real-time traffic involves high bandwidth and it is liable to congestion. Congestion leads to packet losses and bandwidth degradation and also wastes time and energy on congestion recovery.

Mobile Ad Hoc Networks have some unique properties that affect the design of appropriate protocols and congestion control mechanism in particular. As it turned out, the vast different environment in a mobile ad-hoc network is highly problematic for TCP.

The properties of MANETs are the node mobility and a shared, wireless multi-hop channel. Adapting new routes due to node mobility as well as unreliable medium result in unsteady packet delay and packet loses. Also these are not congestion loses.

The wireless multi-hop channel allows only one data transmission at a time within the transmission range of one node. So close links as per the distance are not independent from each other. Internet routers typically are dedicated hosts connected by high bandwidth links. When congestion occurs on the Internet, it is usually concentrated on one single router, but in MANETs congestion affects a whole area because of the shared medium. Not only nodes are overloaded, but regions of the networks are also overloaded.

\section{RELATED WORK}

The continuous research on congestion minimization for MANET still needs more new techniques. This section will illustrate the research related to congestion control. 
International Journal of Wireless \& Mobile Networks (IJWMN) Vol. 4, No. 4, August 2012

A distributed multi-path DSR protocol (MPDSR) was developed to enhance the quality of service [1]. This protocol forwards the outgoing packets using multiple no of paths and with point to point reliability. Split Multi-path Routing protocol [2] uses a per-packet allocation technique to distribute the data packets into multiple paths which prevents nodes from being congested in heavily loaded traffic conditions.

A novel technique Congestion Adaptive Routing in Mobile Ad Hoc Networks (CRP) [3] was proposed. In CRP each node on a route in the network sends a warn message to its previous node when it is to be congested. Then the previous node uses a new route to bypass the congestion and get the first non congested node on the route. Traffic will be distributed probabilistically over these routes and there will be less chance of congestion occurrence.

A dynamic load aware based load-balanced routing for ad hoc networks (DLBL) algorithm was developed. The DLBL [4] uses intermediate node routing load metric that helps the protocol to discover a route with less network congestion. When a link fails because of the node movement the algorithm uses the path maintenance to re join the broken links to get a route from the source to the destination.

Another novel algorithm proposed is a Congestion-aware routing metric (CARM) [5] which considers Mac-overhead, data-rate and buffer queue delay to select low congestion with high throughput routes. Another mechanism that CARM applied was the use of link data- rate categories to prevent the mismatched link data-rate. CARM performed locally.

An ant based DSR [6] will inform the source node about QoS available to destination node such as acceptable delay, jitter and energy in the case of multimedia and real time application. They proposed DSR using ACO and called AntDSR(ADSR).

Another novel algorithm proposed a QoS-aware routing protocol [7] which consider two scheme admission control and feedback for the Qos requirements. Their major works on the approximation of bandwidth estimation to calculate the rest amount of bandwidth available at each node.

A biologically inspired routing algorithm [8] for multi-hop networks considers the concept of stigmergy and evaluates the optimal and sub-optimal routes without the whole system-wide connectivity information. They also incorporate MAC layer information into the routing decision can prevent the forward data traffic into the congested areas under situation like various nodal mobility, network density and data loads.

The success of data transmission in Multipath routing [9] considers the concept of traffic distribution strategy that defines how concurrently available paths are utilized and data to the same destination as split and distributed over multiple paths. This paper shows when the no of path is single the entire data packet is transmitted through it and maximum probability of success as the no of paths increases the probability of success of data transmission will decrease.

\section{PROPOSED WORK}

In this section we will discuss Swarm intelligence, Distance metric and the transmission Queue length.

\subsection{Swarm Behaviour}

This algorithm is inspired by the foraging behaviour of biological ant colony [10], when they find paths to food sources. Each ant deposits a chemical called pheromone when they move from source to the destination and the foragers follow such pheromone trails. By that more ants are attracted by these pheromone trails and in turn reinforce them even more. This natural phenomenon (stigmergy) plays a role in developing and manipulating local information.

\subsection{Congestion Metric}

The major parameters to control congestion areas probabilistically are the two metrics in a node. 


\subsubsection{Distance Metric}

The first metric is the distance between the single hop by using the Time of arrival [11] estimating the time of signal travelling between nodes (TOA) provides the distance between two nodes. We can use ultrasound sensor for measuring range between nodes. For this purpose very accurate rubidium-based oscillators must be used. In order to calculate the TOA parameter the nodes must have a common clock, or exchange timing information by certain protocols such as two-way ranging protocol.

The distance metric $\mathrm{d}_{\mathrm{ij}}$ where $j$ is the neighbor node of $i$.

$$
\mathrm{d}_{\mathrm{ij}}=\mathrm{C} *\left(\mathrm{~d}_{\text {remote }}-\mathrm{d}_{\text {local }}\right) / 2, \quad \mathrm{C} \approx 3 * 10^{8} \mathrm{~m} / \mathrm{s}
$$

The time between starting the transmission of a data packet and receiving the corresponding immediate acknowledge will refer as remote delay $\left(\mathrm{d}_{\text {remote }}\right)$ and receiving one data packet and sending out the immediate acknowledgement will be the duration as local delay $\left(\mathrm{d}_{\text {local }}\right)$. C is measured as the speed of light.

\subsubsection{Transmission Queue Length Metric}

The second metric is a heuristic value transmission queue length in the $i_{\text {th }}$ node towards the neighbouring nodes. The transmission queue length [8] can affect the packet latency and packet drop because of the size of the packet length.

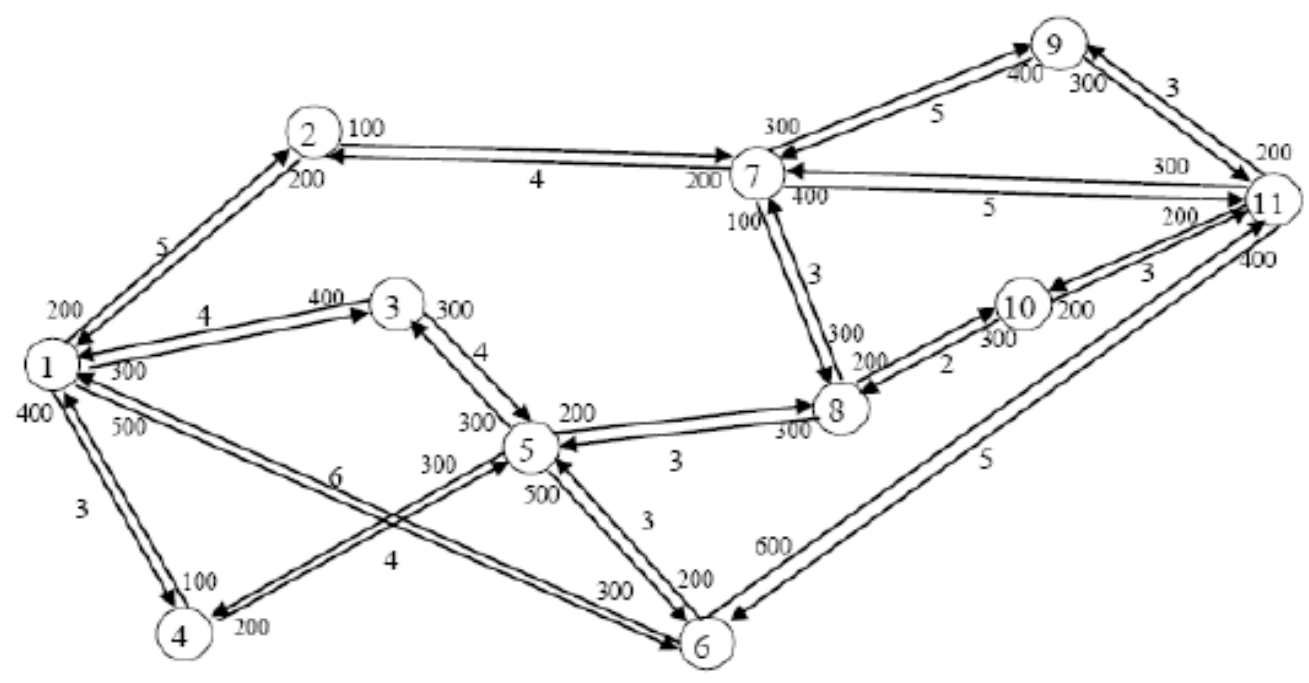

Figure 1: Mobile nodes with distance and out going transmission queue

$$
T_{i, j}=1-\frac{q_{i, j}}{\sum_{j \in N_{b}^{i}} q_{i, j}}
$$


where $q_{i, j}$ is the outgoing queue length in terms of bits between $\mathrm{i}$ and $\mathrm{j}$ and $N$ is all the nodes in the network and $N_{b}{ }^{[i]}$ the node $i$. We have considered some random values as of bits. Using (2), we find the goodness of heuristic values means small queue data length will have higher heuristic value. From the figure 1, all the nodes have symmetric link between them. We consider some hypothetical value as distance and transmission queue length, using (1), we found the congestion metric $T_{i, j}$ in the table 1 [8].

Table 1: Goodness of congestion metric $T_{i, j}$

\begin{tabular}{|l|l|l|l|l|l|l|l|l|l|l|l|}
\hline $\begin{array}{l}j \Rightarrow \\
i \Downarrow\end{array}$ & 1 & 2 & 3 & 4 & 5 & 6 & 7 & 8 & 9 & 10 & 11 \\
\hline 1 & & .85 & .78 & .71 & & .64 & & & & & \\
\hline 2 & .33 & & & & & & .66 & & & & \\
\hline 3 & .42 & & & & .57 & & & & & & \\
\hline 4 & .66 & & & & .33 & & & & & & \\
\hline 5 & & & .76 & .76 & & .61 & & .84 & & & \\
\hline 6 & .66 & & & & .77 & & & & & & .33 \\
\hline 7 & & .80 & & & & & & .90 & .70 & & .66 \\
\hline 8 & & & & & .62 & & .62 & & & .75 & \\
\hline 9 & & & & & & & .42 & & & & .57 \\
\hline 10 & & & & & & & & .40 & & & .60 \\
\hline 11 & & & & & & .63 & .72 & & .81 & .81 & \\
\hline
\end{tabular}

\subsection{Ant based probabilistic path selection}

When a source node $\mathrm{s}$, needs to route a destination d without knowing the global topology, using the initial pheromone information available the ant will chose its next hop with probability $P_{i, j}$. We have proposed an algorithm to find the distance of neighbour node from the $i_{\text {th }}$ node and also we calculate the pheromone deposition, pheromone evaporation and residual pheromone on the link $l_{i, j}$

$$
\Delta \tau_{i j}(t)=m w l_{i, j} * \frac{1}{d_{i, j}}, j \in N_{b}^{[i]}
$$

The distance $d_{i, j}$ from the node $i$ to its neighbouring nodes can be calculated by using the equation 1 , we assume some random distance metric for our algorithm. $m v l_{i, j}$ is the minimum distance selected from the neighbour nodes of $i$. The $\Delta \tau_{i j}(t)$ is the reciprocal of the distance $d_{i, j}$ on the link. We found that the less distance have more reciprocal value of rate of pheromone deposition and vice versa.

$$
\tau_{i j}^{l}(t)=\tau_{i j}(t)+\Delta \tau_{i j}(t) \rho
$$




$$
\tau_{i j}^{e}(t)=\tau_{i j}^{d}(t)(1-\rho)
$$

$\tau_{i j}(t)=0.1$ is the initial pheromone deposition and the $\rho$ [12] specifies the rate at which pheromone evaporates means ant "forget" previous decision for value $\rho=1$ the pheromone evaporates rapidly and search is random, when $\rho=0$ results in slower evaporation rates, $\rho \in[0,1]$. We take $\rho=0.6$. The final pheromone deposition is $\tau^{d}{ }_{i j}(t)$. The final pheromone evaporates from the link is $\tau_{i j}^{e}(t)$. The residual pheromone on the link is.

$$
\tau_{i j}^{r}(t)=\tau_{i j}^{d}(t)-\tau_{i j}^{\rho}(t)
$$

Using (3) : $\Delta \tau_{1,2}(t)=3 * 1 / 5=.6, \Delta \tau_{1,3}(t)=3 * 1 / 4=.75, \Delta \tau_{1,4}(t)=3 * 1 / 3=1$,

$$
\Delta \tau_{1,6}(t)=3 * 1 / 6=.5
$$

Using (4) : $\quad \tau_{1,2}^{d}(t)=.1+\left(.6^{*} .6\right)=.46, \tau_{1,3}^{d}(t)=.1+\left(.75^{*} .6\right)=.55, \tau_{1,4}^{d}(t)=.1+\left(1^{*} .6\right)=.7$,

$$
\tau^{d}{ }_{1,6}(t)=.1+\left(.5^{*} .6\right)=.4
$$

Using (5) : $\quad \tau_{1,2}^{e}(t)=.46^{*}(1-.6)=.18, \tau_{1,3}^{e}(t)=.55^{*}(1-.6)=.22, \tau_{1,4}^{e}(t)=.7 *(1-.6)=.28$,

$$
\tau_{1,6}^{e}(t)=.4 *(1-.6)=.16
$$

Using (6) : $l_{1,2}(t)=.46-.18=.28, l_{1,3}(t)=.55-.22=.33, l_{1,4}(t)=.7-.28=.42$,

$$
l_{1,6}(t)=.4-.16=.24 \text {. }
$$

The link $l_{i, j}$ from node $i$ shows when the distance is less compared to other neighbor nodes, more pheromone deposition occurs. The nodes residual pheromone deposition metric in the table 2.

Table 2: Residual pheromone metric $\tau_{i j}(t)$

\begin{tabular}{|l||l|l|l|l|l|l|l|l|l|l|l|}
\hline $\begin{array}{l}j \Rightarrow \\
i \Downarrow\end{array}$ & 1 & 2 & 3 & 4 & 5 & 6 & 7 & 8 & 9 & 10 & 11 \\
\hline \hline 1 & & .28 & .33 & .42 & & .24 & & & & & \\
\hline 2 & .42 & & & & & & .35 & & & & \\
\hline 3 & .42 & & & & .42 & & & & & & \\
\hline 4 & .42 & & & & .33 & & & & & & \\
\hline 5 & & & .33 & .33 & & .42 & & .42 & & & \\
\hline 6 & .33 & & & & .42 & & & & & & .20 \\
\hline 7 & & .33 & & & & & & .42 & .28 & & .28 \\
\hline 8 & & & & & .30 & & .30 & & & .42 & \\
\hline 9 & & & & & & & .28 & & & & .42 \\
\hline 10 & & & & & & & & .42 & & & .30 \\
\hline 11 & & & & & & .20 & .28 & & .42 & .42 & \\
\hline
\end{tabular}


When the pheromone information are available the ant will chose its next hop with probability $P_{i, j}$. We adopt this concept by using Baye's probability theorem

$$
P_{i, j}(t)=\frac{\left[\tau_{i j}(t)\right]^{\alpha}\left[T_{i j}\right]^{\beta}}{\sum_{j \in N_{b}^{[i]}}\left[\tau_{i j}(t)\right]^{\alpha}\left[T_{i j}\right]^{\beta}}, \quad \text { for } \mathrm{i}=1 \text { to } \mathrm{N}
$$

where $\alpha \geq 0$ is the relative importance of the pheromone trail. $\beta \geq 0$ is the relative importance of the congestion. $\alpha$ and $\beta$ are the two tuneable parameter will control relative weight of pheromone trail and heuristic value $T_{i j}$.

In the following, we calculate the probability corresponding to the node $\mathrm{i} u$ using $\mathrm{j}$.

$$
\begin{aligned}
& P_{1,2}=\left(.28^{3} * .85^{1}\right) /\left(.28^{3} * .85^{1}\right)+\left(.33^{3 *} .78^{1}\right)+\left(.42^{3 *} .71^{1}\right)+\left(.24^{3} .64^{1}\right)=.17 \\
& P_{1,3}=\left(.33^{3} * .78^{1}\right) /\left(.28^{3} * .85^{1}\right)+\left(.33^{3} .78^{1}\right)+\left(.42^{3 *} .71^{1}\right)+\left(.24^{3} * .64^{1}\right)=.25 \\
& P_{1,4}=\left(.42^{3 *} .71^{1}\right) /\left(.28^{3} * .85^{1}\right)+\left(.33^{3} * .78^{1}\right)+\left(.42^{3 *} .71^{1}\right)+\left(.24^{3} * .64^{1}\right)=.48 \\
& P_{1,6}=\left(.24^{3 *} .64^{1}\right) /\left(.28^{3} * .85^{1}\right)+\left(.33^{3 *} .78^{1}\right)+\left(.42^{3 *} .71^{1}\right)+\left(.24^{3} * .64^{1}\right)=.08
\end{aligned}
$$

Analogously, we can calculate the probability corresponding to other nodes which are shown in table 3.

Table 3: Probability metric on $l_{i, j}$

\begin{tabular}{|c||c|c|}
\hline $\mathbf{i}, \mathbf{j}$ & $P_{i, j}(t)$ & $P_{i, j}(t)$ \\
& $\alpha>\beta$ & $\alpha<\beta$ \\
& & \\
\hline \hline 1,2 & .17 & .31 \\
\hline 1,3 & .25 & .28 \\
\hline 1,4 & .48 & .27 \\
\hline 1,6 & .08 & .11 \\
\hline 2,1 & .46 & .13 \\
\hline 2,7 & .53 & .86 \\
\hline 3,1 & .42 & .28 \\
\hline 3,5 & .57 & .71 \\
\hline 4,1 & .80 & .91 \\
\hline 4,5 & .19 & .08 \\
\hline 5,3 & .16 & .22 \\
\hline 5,4 & .16 & .22 \\
\hline 5,6 & .27 & .15 \\
\hline 5,8 & .38 & .39 \\
\hline 6,1 & .28 & .32 \\
\hline 6,5 & .68 & .65 \\
\hline
\end{tabular}

\begin{tabular}{|c||c|c|}
\hline $\mathbf{i}, \mathbf{j}$ & $P_{i, j}(t)$ & $P_{i, j}(t)$ \\
& $\alpha>\beta$ & $\alpha<\beta$ \\
& & \\
\hline \hline 6,11 & .03 & .02 \\
\hline 7,2 & .23 & .26 \\
\hline 7,8 & .53 & .48 \\
\hline 7,9 & .12 & .15 \\
\hline 7,11 & .10 & .09 \\
\hline 8,5 & .18 & .22 \\
\hline 8,7 & .18 & .22 \\
\hline 8,10 & .62 & .55 \\
\hline 9,7 & .17 & .21 \\
\hline 9,11 & .82 & .78 \\
\hline 10,8 & .64 & .29 \\
\hline 10,11 & .35 & .70 \\
\hline 11,6 & .03 & .08 \\
\hline 11,7 & .11 & .17 \\
\hline 11,9 & .42 & .37 \\
\hline 11,10 & .42 & .37 \\
\hline
\end{tabular}


International Journal of Wireless \& Mobile Networks (IJWMN) Vol. 4, No. 4, August 2012

\subsection{Algorithm $P^{2}$ sdt : Node i Processing for distribution of traffic}

Algorithm $\mathrm{P}^{2} \operatorname{sdt}($ )

Input:

$\mathrm{d}_{\mathrm{ij}}$ : Distance between the nodes.

$\mathrm{q}_{\mathrm{ij}}$ : Transmission queue length.

$\alpha$ : Relative importance of pheromone deposition.

$\beta$ : Relative importance of congestion.

$\rho:$ Rate at which pheromone evaporates.

\section{Output:}

$\mathbf{P}_{\mathrm{i}, \mathrm{j}}\left(\tau_{i j}(t)\right)$ : Goodness of Congestion

$\mathrm{P}_{\mathrm{i}, \mathrm{j}}\left(T_{i, j}\right) \quad$ : Residual pheromone

\section{BEGIN}

1. Update parameter for Probabilistic path selection

2. $\tau_{i j}(t) \leftarrow$ Pheromone residual on the link.

3. $T_{i, j} \leftarrow$ Probability of congestion it discards.

4. if $(\alpha>\beta)$ then

5. $\quad$ while $\left(\tau_{i j}(t)>\tau_{i j \in N_{b}}(t)\right)$

6. Distribute.Traffic( $\left.\operatorname{Max}_{\operatorname{Prob}} \operatorname{Pr}_{\mathrm{i}, \mathrm{j}}\left(\tau_{i j}(t)\right)\right)$

7. wend

8. $\quad$ if $\left(\max \left(\tau_{i j}(t)\right)=\tau_{i j \in N_{b}}(t)\right)$ then

9. Distribute.Traffic( $\left.\operatorname{Max}_{\operatorname{Prob}} \mathrm{Pr}_{\mathrm{i}, \mathrm{j}}\left(T_{i, j}\right)\right)$

10. end if

11. else

12. if $(\alpha<\beta)$ then

13. while $\left(T_{i, j}>T_{i, j \in N_{b}}\right)$ then

14. Distribute.Traffic( $\left.\operatorname{Max}_{\operatorname{Prob}} \mathrm{Pr}_{\mathrm{i}, \mathrm{T}}\left(T_{i, j}\right)\right)$

15. wend

16. if $\left(\max \left(T_{i, j}\right)=T_{i, j \in N_{b}}\right)$ then

17. Distribute.Traffic $\left(\operatorname{Max} . P r o b_{\mathrm{i}, \mathrm{j}}\left(\tau_{i j}(t)\right)\right)$

18. end if

19. end if

20. end if

21. END

\subsection{The Route Discovery}

When initialization process starts the single-hop HELLO messages creates the neighbourhood for each node. The HELLO packet contains source IP address and hop count (Initialized to 0). Whenever a traffic source $s$ needs a route to a destination $d$, it broadcasts route request packets (RQ) across the network. The RQ packet contains destination IP address, source IP address, and broadcast ID. For the route discovery the solution would be through flooding the RQ packets, we adopt the probabilistic broadcast scheme in combined with the outgoing queue length $Q_{i, j}$ in terms of bits and the distance metric $D_{i, j}$. When a node first receives a packet, 
International Journal of Wireless \& Mobile Networks (IJWMN) Vol. 4, No. 4, August 2012

with probability $p$ it broadcasts the packet to its neighbours and with probability $1-p$ it discards the packet. The probability value $p$ is calculated as

$$
\begin{aligned}
& Q_{i, j}=\frac{q_{i, j}}{\sum_{j \in N_{b}^{i}} q_{i, j}} \\
& D_{i, j}=\frac{d_{i, j}}{\sum_{j \in N_{b}^{i}} d_{i, j}} \\
& \mathrm{P}_{\mathrm{CONGi,j}}{ }^{(\mathrm{t})}=e^{-\sigma\left(Q_{i, j}\right)} \\
& \mathrm{P}_{\text {Disti,j }}{ }^{(\mathrm{t})}=e^{-\sigma\left(D_{i, j}\right)} \\
& P_{i, j}{ }^{(t)}=\beta\left(\mathrm{P}_{\text {CONGi,j }}{ }^{(\mathrm{t})}\right)+(1-\beta)\left(\mathrm{P}_{\text {Disti,j }}{ }^{(\mathrm{t})}\right),
\end{aligned}
$$

where $\beta$ lies between 0 and 1 .

Table 4: Probability metric of distance and congestion

\begin{tabular}{|c||c|c|}
\hline $\mathbf{i}, \mathbf{j}$ & $\mathrm{P}_{\text {CONGi,j }}$ & $\mathrm{P}_{\text {Disti,j }}$ \\
& & \\
\hline \hline 1,2 & .86 & .76 \\
\hline 1,3 & .80 & .80 \\
\hline 1,4 & .74 & .85 \\
\hline 1,6 & .69 & .71 \\
\hline 2,1 & .51 & .64 \\
\hline 2,7 & .71 & .57 \\
\hline 3,1 & .56 & .60 \\
\hline 3,5 & .65 & .60 \\
\hline 4,1 & .71 & .65 \\
\hline 4,5 & .51 & .56 \\
\hline 5,3 & .78 & .75 \\
\hline 5,4 & .78 & .75 \\
\hline 5,6 & .67 & .81 \\
\hline 5,8 & .85 & .81 \\
\hline 6,1 & .71 & .77 \\
\hline 6,5 & .79 & .81 \\
\hline
\end{tabular}

\begin{tabular}{|l||l|l|}
\hline \multicolumn{1}{|c||}{$\mathbf{i , j}$} & $\mathrm{P}_{\text {CONGi,j }}$ & $\mathrm{P}_{\text {Disti,j }}$ \\
& & \\
\hline 6,11 & .51 & .58 \\
\hline 7,2 & .81 & .79 \\
\hline 7,8 & .90 & .84 \\
\hline 7,9 & .74 & .74 \\
\hline 7,11 & .67 & .74 \\
\hline 8,5 & .68 & .69 \\
\hline 8,7 & .68 & .69 \\
\hline 8,10 & .77 & .77 \\
\hline 9,7 & .55 & .53 \\
\hline 9,11 & .65 & .69 \\
\hline 10,8 & .54 & .67 \\
\hline 10,11 & .67 & .54 \\
\hline 11,6 & .69 & .65 \\
\hline 11,7 & .75 & .77 \\
\hline 11,9 & .82 & .86 \\
\hline 11,10 & .82 & .86 \\
\hline
\end{tabular}


International Journal of Wireless \& Mobile Networks (IJWMN) Vol. 4, No. 4, August 2012

Table 4: Probability metric on $P_{i, j}$

\begin{tabular}{|c||c|c|c|}
\hline $\mathbf{i}, \mathbf{j}$ & $\begin{array}{c}P_{i, j} \\
\beta=.3\end{array}$ & $\begin{array}{c}P_{i, j} \\
\beta=.5\end{array}$ & $\begin{array}{l}P_{i, j} \\
\beta=.7\end{array}$ \\
\hline \hline 1,2 & .79 & .81 & .83 \\
\hline 1,3 & .80 & .80 & .80 \\
\hline 1,4 & .81 & .79 & .77 \\
\hline 1,6 & .70 & .70 & .69 \\
\hline 2,1 & .60 & .57 & .54 \\
\hline 2,7 & .61 & .64 & .66 \\
\hline 3,1 & .58 & .58 & .57 \\
\hline 3,5 & .61 & .62 & .63 \\
\hline 4,1 & .66 & .68 & .69 \\
\hline 4,5 & .54 & .53 & .52 \\
\hline 5,3 & .75 & .76 & .77 \\
\hline 5,4 & .75 & .76 & .77 \\
\hline 5,6 & .76 & .74 & .71 \\
\hline 5,8 & .82 & .83 & .83 \\
\hline 6,1 & .75 & .74 & .72 \\
\hline 6,5 & .80 & .80 & .79 \\
\hline
\end{tabular}

\begin{tabular}{|l||c|c|c|}
\hline \multicolumn{1}{|r|}{$\mathbf{i , j}$} & $\begin{array}{c}P_{i, j} \\
\beta=.3\end{array}$ & $\begin{array}{c}P_{i, j} \\
\beta=.5\end{array}$ & $\begin{array}{l}P_{i, j} \\
\beta=.7\end{array}$ \\
\hline \hline 6,11 & .55 & .54 & .53 \\
\hline 7,2 & .79 & .80 & .80 \\
\hline 7,8 & .85 & .87 & .88 \\
\hline 7,9 & .74 & .74 & .74 \\
\hline 7,11 & .71 & .70 & .69 \\
\hline 8,5 & .68 & .68 & .68 \\
\hline 8,7 & .68 & .68 & .68 \\
\hline 8,10 & .77 & .77 & .77 \\
\hline 9,7 & .53 & .54 & .54 \\
\hline 9,11 & .67 & .67 & .66 \\
\hline 10,8 & .63 & .60 & .57 \\
\hline 10,11 & .57 & .60 & .63 \\
\hline 11,6 & .66 & .67 & .67 \\
\hline 11,7 & .76 & .76 & .75 \\
\hline 11,9 & .84 & .84 & .83 \\
\hline 11,10 & .84 & .84 & .83 \\
\hline
\end{tabular}

\subsection{Algorithm $P^{2} s c m$ : Node i Processing for selection of path}

Algorithm $\mathrm{P}^{2} \mathrm{scm}($ )

\section{Input:}

$\mathrm{d}_{\mathrm{ij}}$ : Distance between the nodes.

$\mathrm{q}_{\mathrm{ij}}$ : Transmission queue length.

\section{Output:}

$\begin{array}{ll}\mathrm{P}_{\text {CONGi,j }}{ }^{(t)} & : \text { Goodness of Congestion } \\ \mathrm{P}_{\text {Disti,j }}\left({ }^{(t)}\right. & : \text { Goodness of distance } \\ P_{i, j}(t) & : \text { Probability of path selection }\end{array}$

\section{BEGIN}

1. Update parameter for Probabilistic path selection

2. $Q_{i, j} \leftarrow$ Relative Pheromone deposition on the link.

3. $D_{i, j} \leftarrow$ Relative congestion on the link

4. $\quad$ if $\left(p_{i j}(t)>p_{i j \in N_{b}}(t)\right)$ then

5. $\quad \operatorname{Max}_{\text {Prob }} \operatorname{Pr}_{\mathrm{i}, \mathrm{j}}\left(p_{i j}(t)\right)$

6. end if

7. END

where $\sigma(\sigma>0)$ is the coefficient. where $\beta \in[0,1]$ is the relative importance of the distance and congestion in the link. When $\beta$ is equal to .5 the probability of goodness of congestion and distance metric can be calculated. This broadcast scheme helps to discover new routes avoiding congestion areas and adopting less distance probabilistically. If the probability value $\mathrm{P}_{\mathrm{CONGi,j}}{ }^{(\mathrm{t})}$ is less means more congestion in the path, due to maximum probability is set on $P_{i, j}{ }^{(t)}$ the other 
International Journal of Wireless \& Mobile Networks (IJWMN) Vol. 4, No. 4, August 2012

parameter like $\mathrm{P}_{\text {Disti,j }}{ }^{(\mathrm{t})}$ must be high means shorter distance is available in the link. When the link have the same probability, this broadcast scheme by changing the tuneable parameter $\beta$ as .7 sets a new probability value to find a new path.

During the course of flooding the intermediate node $j$ receives a Route Request (RQ) packet. By capturing the source address and the previous hop node in the message cache it first set the reverse path to the source. If a valid route to the destination $d$ is available, that is, there is at least one link associated with the pheromone trail greater than the other neighbour node or least distance within the neighbour node, the intermediate node $j$ generates a route reply (RP). The $\mathrm{RP}$ is routed back to the source $s$ via the reverse paths. Otherwise, the RQ is rebroadcast.

When the destination node receives RQ it will send a RP to all the neighbours from which it gets a RQ. The intermediate node $j$ can maintain multiple loop-free paths by recording all the new paths that have the latest sequence number but a lower hop-count in its routing table, and also send a RP to all the neighbours from which it gets a RQ. During the process of the RP back to the source $s$ will assigns an initial pheromone value $\tau_{i, j}{ }^{(t)}$ to the corresponding neighbour node, which indicates a valid route to the destination $d$.

\subsection{Description of the Algorithms}

Our proposed $\mathrm{p}^{2} \mathrm{scm}$ algorithm presents the pseudo code of how a node $i$ process the out going queue and by using swarm intelligence how it will select the path and based on the routing table, the $\mathrm{p}^{2}$ sdt will distribute the data traffic according to the probabilities for each neighbour node $\mathrm{j}$ in the routing table[12].

The node $i$ first calculate the distance $\mathrm{d}_{\mathrm{ij}}$ between all its neighbour nodes. By using the smallest distance $m v l_{i, j}$ the node $i$ evaluate the relative distance between all its neighbour nodes. $\mathrm{P}^{2}$ sdt is also evaluate the $\Delta \tau_{i j}(t)$ which is reciprocal to the distance $\mathrm{d}_{\mathrm{ij}}$ where the relative pheromone deposition occurs on the link $l_{i, j}$. Now $\mathrm{p}^{2}$ sdt evaluate the residual pheromone on the link $l_{i, j}$ from the line 2 of the above algorithm. Secondly $\mathrm{p}^{2}$ sdt evaluate the probability of goodness of congestion $\left(1-\frac{q_{i, j}}{\sum_{j \in N_{b}^{[i]}} q_{i, j}}\right)$.

In our proposed algorithm from line no 4-10 when $\alpha>\beta$ it first checks the pheromone $\left(\tau_{i j}(t)\right)$ deposition in a particular link have the highest value within its neighbour or not. If found yes then the maximum probability $\mathrm{P}_{\mathrm{i}, \mathrm{j}}$ will be on that link $l_{i, j}$. Next it checks a pair of paths with highest deposition and same value, if found yes within that, less congested $\left(\mathrm{T}_{\mathrm{i}, \mathrm{j}}\right)$ path will have the highest probability[13].

Similarly from line no $12-20$ when the $\alpha<\beta$, if $T_{i, j}$ have the highest value within its neighbour then the highest probability will be on that link $l_{i, j}$. Next it checks a pair of paths with highest and same value of $T_{i, j}$ or not if found yes within that, more pheromone $\left(\tau_{i j}(t)\right)$ deposited path will have the highest probability on that link $l_{i, j}$.

\section{ANALYSIS}

$\mathrm{P}^{2}$ sdt at first from node $i$ and the two tuneable parameter $\alpha>\beta$ we observe the higher pheromone on the link $l_{i j}$ will have higher probability of path selection and higher goodness of congestion means small queue data length will have low probability, if the deposition is same on both the link, we observe then $\mathrm{p}^{2}$ sdt by using $\alpha>\beta$ and conditional probability evaluates the less congested path means queue data length is small. In our example node 3 to its neighbour node 1 and 2 from table 1 , table 2 and table 3 .

Also by making $\alpha<\beta \mathrm{p}^{2}$ sdt consider the less congested path probabilistically means when the queue data length is same on both the link, the higher probability comes with higher pheromone 
International Journal of Wireless \& Mobile Networks (IJWMN) Vol. 4, No. 4, August 2012

concentration on the link and if the congestion is same within a pair of paths then it evaluates the paths which have more pheromone deposition.

By using two tuneable parameters $\mathrm{p}^{2}$ sdt decides the path probabilistically which have less congested or more pheromone deposition when the nodes have dynamic topology at real time situation.

Similarly $\mathrm{P}^{2} \mathrm{scm}$ evaluates the goodness of congestion as well as distance. If $p_{i j}(t)>$ $p_{i j \in N_{b}}(t)$ means the average value of $p_{i j}(t)$ is maximum within all the links of the neighbouring nodes. Another important feature of $\mathrm{P}^{2} \mathrm{scm}$ is that if the probabilistic value of goodness of congestion is low, the probabilistic value of goodness of distance must be high. As we have seen from the table 4 .

\section{CONCLUSION}

In this paper, we propose a new mathematical model for congestion control. We have developed a single hop congestion aware probabilistic path selection algorithm which employs the swarm intelligence of biological ant. Using distance and congestion metric the algorithm invokes route discovery process with less congestion and shorter distance in a single hop at run time. By probabilistic approach the algorithm can prevent forward data traffic into the congested areas and also finds the shortest distance probabilistically within a pair of nodes. For data transmission, if more congestion occurs in all the links of a node, the algorithms by using the tuneable parameter that can shift the mode of transmission and can easily avoid the congested areas. By using the above stated mathematical models and some hypothetical data we tried to control the congestion at local node level.

\section{REFERENCES}

[1] R.Leung, Jilei Liu, E.Poon, C.A.Chan \& Baochun Li, (2001) "MPDSR : a QoS-aware multipath dynamic source routing protocol for wireless ad-hoc networks", In proceedings Local Computer Networks, $26^{\text {th }}$ Annual IEEE Conference, pp 132-141.

[2] S.Lee \& Mario Garla, (2001) "Split Multi-path Routing with Maximally Disjoint Paths in Ad Hoc Networks", In proceedings International IEEE Conference on Communication, pp 32013205.

[3] Duc. A.Tran \& Harish Raghavendra, (2006) “Congestion Adaptive Routing in Mobile Ad Hoc Networks", IEEE transaction on parallel and Distributed systems, 17: pp 1294-1305.

[4] Xiangquan Zheng, Wei Guo, R.Liu \& Yongchun Trian, (2004) “ A New Dynamic Load-aware Based Load-balanced Routing for Ad Hoc Networks”, IEEE, pp 407-411.

[5] Xiaoqin Chen, H.M.Jones \& A.D.S.Jatyalath, (2007) "Congestion- Aware Routing Protocol for Mobile Ad Hoc Networks", In proceedings of IEEE conference on vehicular Technology, Doi.10.1109/VETECF.2007.21, pp 21-25.

[6] R.Asokan, A.M.Natarajan \& C.Venkatesh, (2008) "Ant Based Dynamic Source Routing Protocol to Support Multiple Quality of Service(QoS) Metrics in Mobile Ad Hoc Networks", International Journal of Computer Science and Security, vol.2 no.3, pp 48-56.

[7] L. Chen \& Wendi B. Heinzelman, (2005) "Qos-Aware Routing Based on Bandwidth Estimation for Mobile Ad Hoc Networks”, IEEE on Selected Areas in Communication, vol.23, pp 561-572.

[8] L. Zhenyu, M.Z.Kwiatkowska \& Costas Constantinou, (2005) “A Biologically Inspired Congestion Control Routing algorithm For MANETs", In Proceedings of the Third IEEE International Conference on Pervasive Computing and Communications , pp 226-231. 
International Journal of Wireless \& Mobile Networks (IJWMN) Vol. 4, No. 4, August 2012

[9] A. K. Mondal \& P. K. Banerjee, (2008) " The Success of data Transmission in Multipath Routing for MANET”, Mobile and Pervasive Computing, CoMPC-2008, pp 48- 51.

[10] R. Beckers, J.L.Deneubourg \& S.Goss, (1992) "Trails and u turns in the selection of the shortest path by the ant lasius niger", Journal of Theoretical Biology, pp 397-415.

[11] A. Gunther \& Christian hoene, (2005) "Measuring Round trip times to determine the distance between WLAN nodes", In proceedings of Networking 2005, Springer-Verlag, pp 768-779

[12] A.C.S.Jawahar, "Ant Colony Optimization for Mobile Ad-Hoc Networks", ajaychak@eden.rutgers.edu.

[13] S. Soardar, V. Bhattacherjee \& D. Giri, (2012) “ A Swarm inspired Probabilistic Path Selection with Congestion Control in MANETs “, In Proceedings of second international conference on Computer Science, Engineering and Application, Springer-Verlag, Vol.2, pp 617-626.

\begin{abstract}
Authors
Subhankar Joardar is currently working as Assistant Professor at Haldia Institute of Technology, Haldia. He completed his MCA and M. Tech from Birla Institute of Technology, Mesra. Currently he is pursuing PhD From BIT, Mesra. His current research areas are Mobile ad Hoc Networks, Swarm Intelligence and Advanced Algorithm design.
\end{abstract}

DR. Vandana Bhattacherjee is a member of the IEEE and the IEEE Computer Society. She is currently working as Associate Professor at Birla Institute of Technology, Ranchi. She completed her B. E. in Computer Science and Engineering from BIT Mesra in 1989, and M. Tech and PhD from Jawaharlal Nehru University, New Delhi in 1991 and 1995 respectively. Her current research areas are Software Process Models, Software Cost Estimation, Data Mining and Mobile ad Hoc Networks.
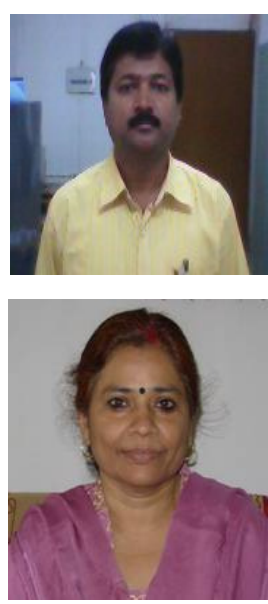

Dr. Debasis Giri is presently Professor in the Department of Computer Science and Engineering, Haldia Institute of Technology, Haldia-721657, India. He received his Ph.D degree from Indian Institute of Technology, Kharagpur 721 302, India 1n 2009. He did his masters (M. Tech and M. Sc) both from IIT, Kharagpur in 2001 and 1998 respectively. He has tenth All India Rank with percentile score 98.42 in the Graduate Aptitude Test in Engineering (GATE) Examination in 1999. He received certificate from All India Science Teachers' Association for success in Science Aptitude \& Talent Search Test in 1988. He has published more than 20 technical papers in the referred journals/conferences. He is presently Editorial

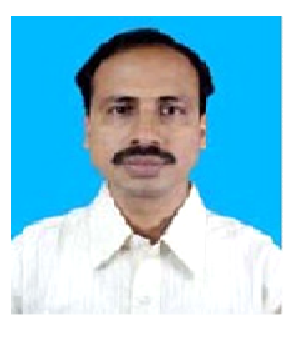
Board Members of many reputed Journals, namely Journal of Convergence, International Journal of Computers and Applications, Journal of Security and Communication Networks (Wiley InterScience) etc. Further, he is a Reviewer of many reputed International Journals. He is also Program Committee member of many International Conferences. He is a Life member of Cryptology Research Society of India. His current research interests include cryptography, Network security, Information security, Ecommerce security, Security in Wireless Sensor Networks, Security in VANETs and Wireless Networks. 